\title{
A Case of Cerebral Amyloid Angiopathy Presented with Cognitive Decline and Hoarding Behavior
}

\author{
Jeewon Lee1, Soyoung Irene Lee1, Shin Gyeom Kim¹, Jungbin Lee², and Han Yong Jung ${ }^{\circledR}$ \\ 1'Department of Psychiatry, Soonchunhyang University Bucheon Hospital, Bucheon, Republic of Korea \\ ${ }^{2}$ Department of Radiology, Soonchunhyang University Bucheon Hospital, Bucheon, Republic of Korea
}

Dear Editor,

Here we report a case of an elderly patient with progress cognitive impairment and hoarding behavior who was diagnosed with cerebral amyloid angiopathy. The study was approved by the Institutional Review Board (IRB) of Soonchunhyang University Bucheon Hospital (2019-11-020).

A 75-year-old female patient visited the outpatient clinic presented with a 1-year history of cognitive decline and hoarding behavior. She was an elementary school graduate and a widow who used to live alone. When her mother passed away a year ago, she had a period of bereavement. Around that time, she started to have trouble remembering the location of her possessions. Her memory impairment rapidly worsened over time and deficits in instrumental activities of her daily living performance became prominent over the past several months. A month ago, she had to move in with her daughter and her disorientation to time and person was worsened to a degree which she became difficult to recognize her sister or her sonin-law. Her hoarding behavior also had developed over the past year. She had difficulty discarding objects and compulsively collected various items that were no value to her and accumulated in her home. When her daughter cleared the patient's house a month ago, every surface in her home except for where she slept and ate was covered with stacks of items.

During the interview, she showed euthymic mood and appropriate range of affect. She showed concerns of her cognitive impairment but lacked awareness of her problem of hoarding.

Received: December 11, 2019 Revised: February 12, 2020

Accepted: February 16, 2020

$\triangle$ Correspondence: Han Yong Jung, MD, PhD

Department of Psychiatry, Soonchunhyang University Bucheon Hospital, 170

Jomaru-ro, Bucheon 14584, Republic of Korea

Tel: +82-32-621-5063, Fax: +82-32-621-6950

E-mail: hanyjung@schmc.ac.kr

(a) This is an Open Access article distributed under the terms of the Creative Commons Attribution Non-Commercial License (https://creativecommons.org/licenses/bync/4.0) which permits unrestricted non-commercial use, distribution, and reproduction in any medium, provided the original work is properly cited.
She had terminal insomnia which have developed over the past several months.

She had no history of head trauma, alcohol abuse, or any psychiatric disease. She was taking Loxoprofen (non-steroidal anti-inflammatory drug, NSAID) for her back pain due to herniated nucleus pulposus. Laboratory screening was normal. Her neurological examination revealed no neurological deficit.

Neurocognitive assessment revealed a Mimi Mental State Examination (MMSE) score of 12/30, a Clinical Dementia Rating (CDR) scale of 1, and a Clinical Dementia Rating-Sum of Box score of 5. She had fluent speech without semantic deficits, but her attention, recall, registration and orientation showed impairment. She had poor executive function and mild ideomotor apraxia on performing commands.

Magnetic resonance imaging (MRI) of the brain demonstrated lobar-distributed multiple cortical-subcortical microhemorrhages in the right temporal lobe (Figure 1). Gyriform dark signal intensity were noted along the right parietal cortical sulci, which indicate superficial cortical siderosis. Additionally, mild to moderate small vessel disease in the bilateral cerebral deep white matter with old lacunar infarctions in the left basal ganglia was found. Mild diffuse brain atrophy, more predominant in the right anterio-temporal lobe was also noted.

According to the Boston Criteria for the diagnosis for CAA, the patient was diagnosed with probable CAA. ${ }^{1}$ Donepezil 5 mg was prescribed for her cognitive impairment. Anticoagulation therapy was not recommended. The NSAID she was taking for her back pain was replaced with acetaminophen due to the high bleeding tendency of the NSAID. She had a blood pressure of 140/90, which would have not usually needed treatment, ${ }^{2}$ but to lower the risk of ICH, hypertensive agent was prescribed for strict management of her blood pressure.

After discharge, the patient complained of nausea due to donepezil, so it was switched to galantamine. Galantamine also showed gastrointestinal side effects while her cognitive dysfunction became worse. Galantamine was changed to 

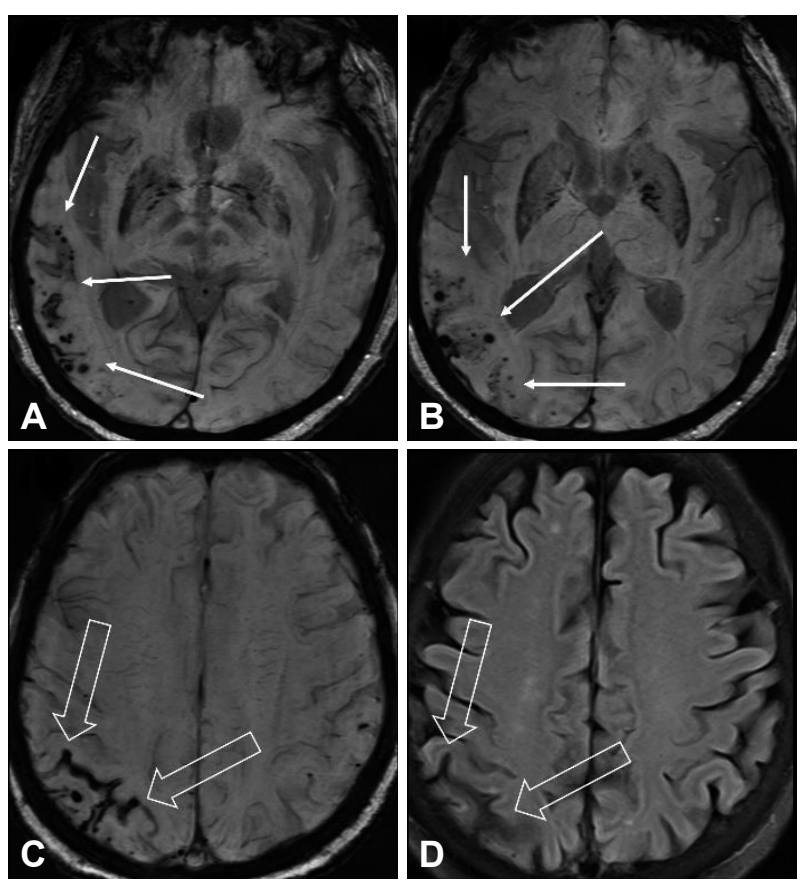

Figure 1. Brain magnetic resonance imaging of the patient. A, B: Susceptibility-weighted imaging (SWI) demonstrated lobar-distributed multiple cortical-subcortical microhemorrhages in the right temporal lobe (arrows). C, D: On T2-weighted fluid-attenuated inversion recovery (FLAIR) and SWI, gyriform dark signal intensity (open arrows) were noted along the right parietal cortical sulci, which indicate superficial cortical siderosis.

memantine and quetiapine was added for her recently aggravated symptoms of sleep disturbance and perceptual disturbance. Her cognitive function and disability in basic activities of daily living have been deteriorating rather rapidly after her diagnosis. Her daughter who is currently living with the patient have precluded her from collecting items.

Cerebral Amyloid Angiopathy (CAA) is a clinicopathological entity characterized by beta-amyloid peptide deposits within blood vessels of the brain and leptomeninges. ${ }^{3}$ Amyloid deposition within the media and adventitia of cerebral arteries lead to smooth muscle cell destruction, vascular wall thickening, vessel fragility and rupture. ${ }^{4}$ Lobar microbleeds are the hallmark of CAA and occur more frequently in the temporal and occipital lobes. ${ }^{3}$ Also, lobar microbleeds tend to cluster in the same lobe when there are multiple lesions. Patients with CAA have high risk of recurred microbleeds. A study showed that $50 \%$ of patients with CAA developed new, frequently multiple microbleeds after 15 months. ${ }^{5}$

Progressive cognitive impairment in the elderly is closely associated with CAA. CAA is found in $75-90 \%$ of Alzheimer's disease $(\mathrm{AD})$ cases, and advanced CAA is present in approximately $25 \%$ of $\mathrm{AD}$ cases. ${ }^{6}$ Lobar microbleeds are more prevalent in $\mathrm{AD}$ compared to many other causes of dementia. ${ }^{3} \mathrm{CAA}$ accompanied by $\mathrm{AD}$ may produce more severe cognitive dys- function. ${ }^{3}$ However, it has been reported that CAA pathology itself is an independent contributor to late-life cognitive outcomes. ${ }^{7}$ CAA pathology has been reported to be associated with rapid decline in global cognition, perceptual speed, episodic and semantic memory.

Many studies have demonstrated the development of hoarding behaviors in relation to dementia. ${ }^{8}$ It has been reported that hoarding behaviors were found in $22.6 \%$ of dementia patients admitted in a geriatric ward. ${ }^{9}$ Dysfunction of the frontolimbic-striatal system, predominantly in the right, is discussed to be associated with compulsive hoarding behaviors. ${ }^{10}$ Cognitive dysfunction, particularly problems in executive functioning including decision-making categorization, organization, and planning, has been suggested as a vulnerable factor for the development of hoarding behaviors. ${ }^{8}$ Also, deficits in information processing resulting in erroneous beliefs about the nature of objects may also contribute in hoarding behaviors.

The present case demonstrates the wide spectrum of clinical manifestation of CAA. Hoarding behavior is not a clinical symptom which have been reported to be associated with CAA. CAA may have a varied presentation complicating the differential diagnosis of dementia. Akers et al. ${ }^{11}$ reported series of CAA cases with primary progressive aphasia, normal pressure hydrocephalus, and Lewy body dementia, which are also not typically thought to be associated with CAA. The presence of CAA in the context of coexisting multiple neurodegenerative syndromes is a significant variable. Identifying dementia patients with accompanying CAA is especially important because it can have critical therapeutic consequences regarding anticoagulation therapy, blood pressure control, and prognosis due to its high risk of spontaneous brain hemorrhages. ${ }^{3}$ Early identification of CAA in older adults before they experience a devastating lobar ICH or cognitive impairment is necessary, and understanding the wide spectrum of clinical manifestation of CAA would be important.

\section{Acknowledgments}

This study was supported by the Soonchunhyang Research Fund.

\section{Conflicts of Interest}

The authors have no potential conflicts of interest to disclose.

\section{ORCID iDs}

Jeewon Lee

Soyoung Irene Lee

Shin Gyeom Kim

Jungbin Lee

Han Yong Jung

https://orcid.org/0000-0001-5930-1834 https://orcid.org/0000-0003-2473-2954 https://orcid.org/0000-0001-8196-655X https://orcid.org/0000-0002-6240-8277 https://orcid.org/0000-0003-0089-0368

\section{REFERENCES}

1. Knudsen KA, Rosand J, Karluk D, Greenberg SM. Clinical diagnosis of cerebral amyloid angiopathy: validation of the Boston criteria. Neurology 2001;56:537-539. 
2. Kovell LC, Ahmed HM, Misra S, Whelton SP, Prokopowicz GP, Blumenthal RS, et al. US hypertension management guidelines: a review of the recent past and recommendations for the future. J Am Heart Assoc 2015;4. pii: e002315.

3. Viswanathan A, Greenberg SM. Cerebral amyloid angiopathy in the elderly. Ann Neurol 2011;70:871-880.

4. Vinters HV. Cerebral amyloid angiopathy. A critical review. Stroke 1987;18:311-324.

5. Greenberg SM, Eng JA, Ning M, Smith EE, Rosand J. Hemorrhage burden predicts recurrent intracerebral hemorrhage after lobar hemorrhage. Stroke 2004;35:1415-1420.

6. Jellinger KJ. Alzheimer disease and cerebrovascular pathology: an update. J Neural Transm (Vienna) 2002;109:813-836.
7. Boyle PA, Yu L, Nag S, Leurgans S, Wilson RS, Bennett DA, et al. Cerebral amyloid angiopathy and cognitive outcomes in community-based older persons. Neurology 2015;85:1930-1936.

8. Ayers CR, Najmi S, Mayes TL, Dozier ME. Hoarding disorder in older adulthood. Am J Geriatr Psychiatry 2015;23:416-422.

9. Hwang JP, Tsai SJ, Yang CH, Liu KM, Lirng JF. Hoarding behavior in dementia: A preliminary report. Am J Geriatr Psychiatry 1998;6:285289 .

10. Finney CM, Mendez MF. Diogenes syndrome in frontotemporal dementia. Am J Alzheimers Dis Other Demen 2017;32:438-443.

11. Akers C, Acosta LM, Considine C, Claassen D, Kirshner H, Schrag MJ, et al. Atypical clinical manifestations of cerebral amyloid angiopathy. Curr Neurol Neurosci Rep 2019;19:64. 